IUCrJ

ISSN 2052-2525

BIOLOGY|MEDICINE

Received 13 June 2019

Accepted 5 September 2019

Edited by J. Trewhella, University of Sydney, Australia

Keywords: polyketide biosynthesis; antibiotics; Steptomyces antibioticus; cycloaddition; crystal structure; protein crystallography; QM/MM reaction modelling; Diels-Alder reaction.

PDB references: IdmH, truncated ( $\Delta 99-107)$, selenomethionine-containing, $6 \mathrm{hnl}$; truncated (499-107), wild type, 6hnm; full length, wild type, 6hnn

Supporting information: this article has supporting information at www.iucrj.org

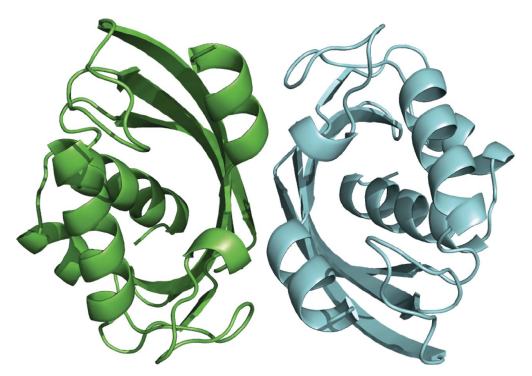

OPEN Ә ACCESS

\section{Crystal structure of the putative cyclase IdmH from the indanomycin nonribosomal peptide synthase/ polyketide synthase}

\author{
leva Drulyte, Jana Obajdin, ${ }^{a}$ Chi H. Trinh,, ${ }^{\text {a }}$ Arnout P. Kalverda, ${ }^{a}$ Marc W. van der

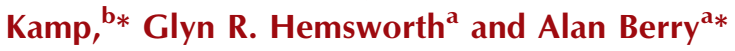

\begin{abstract}
aAstbury Centre for Structural Molecular Biology and School of Molecular and Cellular Biology, University of Leeds, Leeds LS2 9JT, England, and ${ }^{\mathbf{b}}$ School of Biochemistry, University of Bristol, University Walk, Bristol BS8 1TD, England.

*Correspondence e-mail: marc.vanderkamp@bristol.ac.uk, a.berry@leeds.ac.uk
\end{abstract}

Indanomycin is biosynthesized by a hybrid nonribosomal peptide synthase/ polyketide synthase (NRPS/PKS) followed by a number of 'tailoring' steps to form the two ring systems that are present in the mature product. It had previously been hypothesized that the indane ring of indanomycin was formed by the action of IdmH using a Diels-Alder reaction. Here, the crystal structure of a selenomethionine-labelled truncated form of IdmH (IdmH- $\Delta$ 99-107) was solved using single-wavelength anomalous dispersion (SAD) phasing. This truncated variant allows consistent and easy crystallization, but importantly the structure was used as a search model in molecular replacement, allowing the full-length IdmH structure to be determined to $2.7 \AA$ resolution. IdmH is a homodimer, with the individual protomers consisting of an $\alpha+\beta$ barrel. Each protomer contains a deep hydrophobic pocket which is proposed to constitute the active site of the enzyme. To investigate the reaction catalysed by $\mathrm{IdmH}$, $88 \%$ of the backbone NMR resonances were assigned, and using chemical shift perturbation of $\left[{ }^{15} \mathrm{~N}\right]$-labelled $\mathrm{IdmH}$ it was demonstrated that indanomycin binds in the active-site pocket. Finally, combined quantum mechanical/ molecular mechanical (QM/MM) modelling of the IdmH reaction shows that the active site of the enzyme provides an appropriate environment to promote indane-ring formation, supporting the assignment of IdmH as the key DielsAlderase catalysing the final step in the biosynthesis of indanomycin through a similar mechanism to other recently characterized Diels-Alderases involved in polyketide-tailoring reactions. An animated Interactive 3D Complement (I3DC) is available in Proteopedia at https://proteopedia.org/w/Journal: IUCrJ:S2052252519012399.

\section{Introduction}

Polyketides and nonribosomal peptides are two major classes of natural products which give rise to nearly one third of the current pharmacopoeia (Newman \& Cragg, 2012). They exhibit high structural diversity and are proven to be excellent therapeutics, but there is an increasing interest in diversifying current natural product libraries to produce analogues with improved or novel biological activity (Cummings et al., 2014). Natural products classed as polyketides, nonribosomal peptides and hybrids of both are often biosynthesized by giant, complex enzymes known as polyketide synthases (PKS) and nonribosomal peptide synthetases (NRPS). Both systems consist of a range of domains which incorporate a number of starter and extender units to build a linear structure. In the case of polyketides, the linear enzyme products can then be further tailored by various enzymes, including cyclases, oxidases, reductases and methylases, to yield a diverse array of bioactive compounds (Olano et al., 2010). 
Indanomycin (1) is an antibiotic from the pyrroloketoindane family which is known to act as an effective ionophoric agent against Gram-positive bacteria (Dutton et al., 1995). Indanomycin is synthesized in Streptomyces antibioticus NRRL 8167 by a hybrid nonribosomal peptide synthetase/ polyketide synthase (NRPS/PKS; Li et al., 2009). The NRPS portion of the pathway is proposed to generate a pyrrole moiety from L-proline, which is then extended by the sequential addition of malonyl-CoA, methylmalonyl-CoA and ethylmalonyl-CoA building blocks by ten predicted PKS modules to yield the linear nonribosomal peptide-polyketide hybrid natural product 2 (Fig. 1). At least two cyclization reactions are then needed to generate the tetrahydropyran and tetrahydroindane rings of indanomycin (1; Fig. 1). The former could be installed while the polyketide is still tethered to the PKS by the cyclase domain, Cyc11, in the terminal, 11th PKS module. Indane-ring formation has been postulated to be catalysed by a separate indane cyclase, IdmH (Li et al., 2009; Rommel et al., 2011), using a Diels-Alder [4+2] intramolecular cyclization (Fig. 1). The feasibility of indane-ring formation in indanomycin proceeding via a Diels-Alder reaction was first shown in its complete chemical synthesis (Edwards et al., 1984), but to date it has not been possible to characterize the activity of IdmH in molecular detail.
The proposed biosynthetic cycloaddition would require the appropriate diene and dienophile within the intermediate. The indanomycin PKS harbours catalytic domains which would produce the suitable diene intermediate for the cycloaddition to be feasible. However, the required dienophile would not be generated by straightforward use of the catalytic domains present in the indanomycin PKS/NRPS, since the second PKS module is predicted to install a hydroxyl group at C19 (Fig. 1, compound 2; Rommel et al., 2011). To form the appropriate dienophile, dehydration of the alcohol at C19 is therefore needed, either while the linear polyketide is tethered to the synthase or after it has been released. One suggested possibility is that a dehydratase (DH) domain, for example DH3, from a neighbouring module might catalyse the required dehydration in a manner similar to that used in epothilone biosynthesis (Tang et al., 2004; Li et al., 2009).

Irrespective of the activity required to dehydrate C19, IdmH has been proposed to catalyse the Diels-Alder reaction which would lead to the final indane-ring formation in indanomycin. In support of this idea, an $i d m H$ deletion mutant of $S$. antibioticus exhibited both a significant reduction in indanomycin yield and the production of a previously unobserved linear tetraene alternative product (Rommel et al., 2011). Notably, indanomycin-production levels were restored when
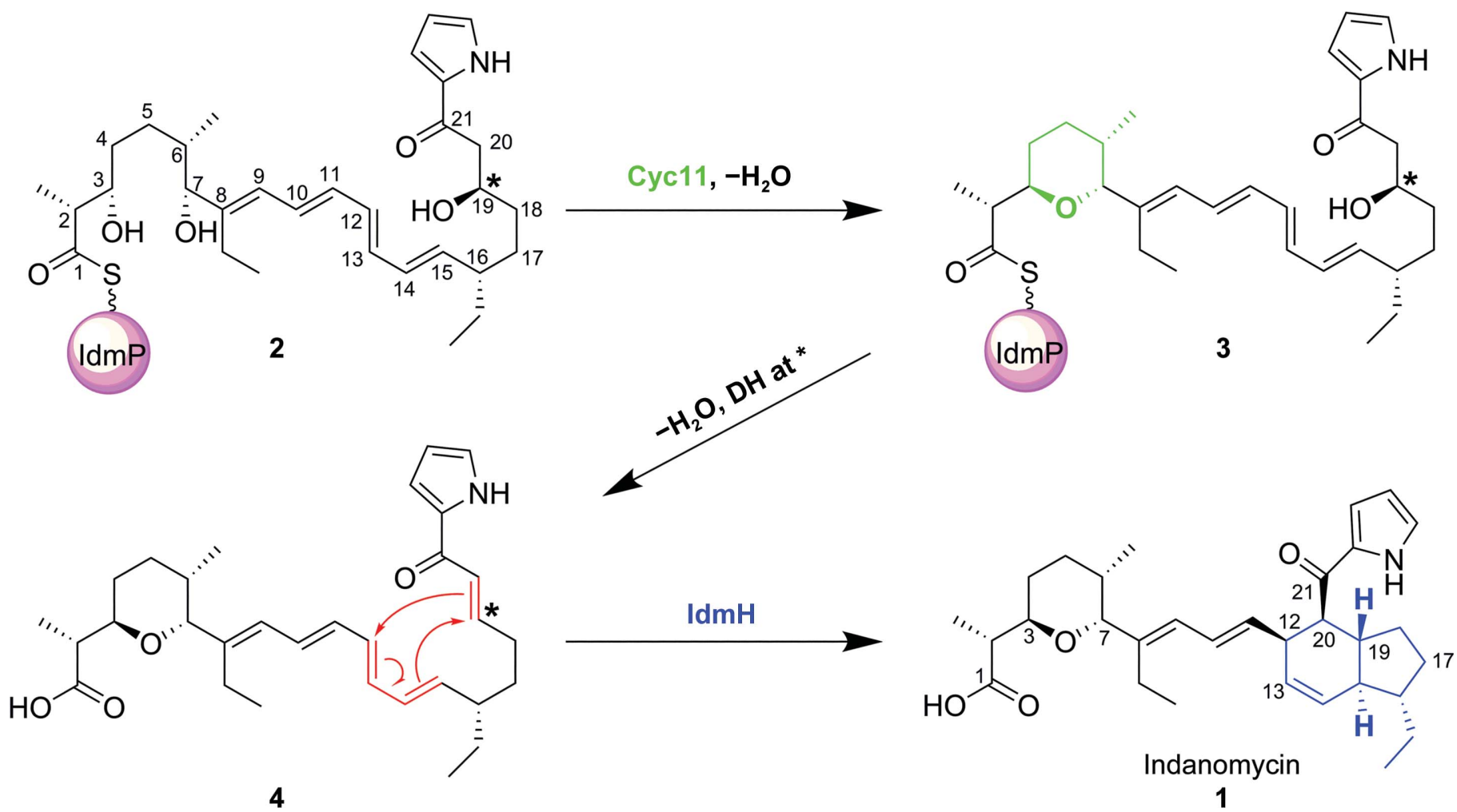

Figure 1

The proposed mechanism for indanomycin maturation through the formation of tetrahydropyran (green) and indane (blue) rings. After 'starter' pyrrole biosynthesis, the polyketide is built through the actions of the five Idm ORFs IdmL-P, and the final polyketide chain is left attached to IdmP. Cyc11 is thought to mediate the formation of the tetrahydropyran ring through a direct nucleophilic replacement to generate $\mathbf{3}$ (Li et al., 2009). This reaction could then be followed by hydrolysis to release $\mathbf{4}$ from the IdmP subunit. Finally, indane-ring formation is thought to be mediated by a putative cyclase, IdmH (Li et al., 2009). For [4+2] cycloaddition to occur, C19 of $\mathbf{2}$ (denoted with an asterisk) needs to be dehydrated to produce a double bond to act as the dienophile (Rommel et al., 2011). Since the second PKS module does not contain a dehydratase domain, a hypothetical dehydration step was included between intermediates 3 and $\mathbf{4}$. Adapted from Li et al. (2009). 
the $i d m H$ gene was introduced to $S$. antibioticus in trans (Rommel et al., 2011). IdmH is thus thought to be the key cyclase responsible for the formation of the indane ring and hence mature indanomycin. $\mathrm{IdmH}$ is a protein consisting of 145 amino acids (Supplementary Fig. S1) exhibiting sequence similarity to a number of natural product-modifying enzymes. These include the epoxide hydroxylase MonBI from S. cinnamonensis $(50 \%$ identity over $14 \%$ of the sequence; Minami et al., 2014) and the polyketide cyclase SnoaL from S. nogalater (27\% identity over $91 \%$ of the sequence; Sultana et al., 2004) (Supplementary Fig. S2). Both homologues exhibit an $\alpha+\beta$ barrel fold and are involved in ring closure and aromatic ring hydroxylations in the monensin and nogalamycin biosynthetic pathways, respectively. Beyond polyketidemodifying enzymes, other $\alpha+\beta$ barrel proteins include $\Delta^{5}-3$ ketosteroid isomerase and scylatone dehydratase (Ha et al., 2001; Lundqvist et al., 1994), showing that nature has made broad use of this fold in many biosynthetic pathways. Given the diversity of reactions catalysed by enzymes displaying this fold, it is challenging to predict the function of IdmH based on sequence similarity alone.

As a step towards elucidating the underlying enzymatic mechanism of indanomycin maturation, we set out to determine the three-dimensional structure of IdmH. Here, we present crystal structures of $\operatorname{IdmH}$ and a deletion variant, IdmH- $\Delta 99-107$, at 2.7 and $2 \AA$ resolution, respectively. Structural analysis shows that IdmH has a similar fold to that of the putative hydroxylases SnoAL2 and AcIR (Beinker et al., 2006) and the polyketide cyclases SnoaL (Sultana et al., 2004) and AknH (Kallio et al., 2006). Apparent differences in the putative active site, however, suggest that this enzyme catalyses a distinct reaction from that of its closest structural homologues. We therefore utilized NMR spectroscopy and in silico modelling [including quantum mechanical/molecular mechanical (QM/MM) reaction simulations] to probe product binding and the likely reaction catalysed by $\mathrm{IdmH}$, with our analyses supporting the notion that this enzyme is indeed a Diels-Alderase.

\section{Materials and methods}

\subsection{Plasmid construction}

The gene sequence encoding the full-length $i d m H$ gene (ACN69984.1) was amplified by PCR using the genomic DNA from S. antibioticus (strain NRRL 8167) as a template, and the forward and reverse primers 5 -ctg gtg ccg cgc ggc agc cat ATG GCT CAT CAG CCT TCG- $3^{\prime}$ and $5^{\prime}$-tcc acc agt cat get agc caT CAC AGG GAC GCC TTC AC-3', respectively. The amplified sequence contained overhang sequences (in lower case letters) which facilitated ligation to an NdeI-linearized pET-28(a)+ vector using the NEBuilder HiFi DNA-assembly kit (NEB). The generated plasmid also encoded an N-terminal hexahistidine tag on the protein and a thrombin cleavage site. The gene insert was confirmed by sequencing with $\mathrm{T} 7$ and T7term universal primers before transformation into Escherichia coli strain BL21(DE3) cells for overexpression. Site-directed mutagenesis to generate the deletion variant IdmH- $\Delta 99-107$ was performed using wild-type pET-28_IdmH as a template and the forward and reverse primers 5'-CGC GAC CGC GAG GGG TGG-3' and 5'-GGA GTG CGT TCC CCG TGC-3', respectively. The site-directed mutagenesis PCR product was treated with KLD Enzyme Mix (NEB) before transformation into $E$. coli strain 5- $\alpha$ competent cells.

\subsection{Expression and purification of $\mathrm{IdmH}$}

Recombinant IdmH was expressed in E. coli strain BL21(DE3) cells harbouring the pET-28_IdmH plasmid. The cells were grown in $2 \mathrm{TY}$ medium containing $50 \mu \mathrm{g} \mathrm{ml}^{-1}$ kanamycin at $37^{\circ} \mathrm{C}$ to an $\mathrm{OD}_{600}$ of 0.4 before lowering the temperature to $15^{\circ} \mathrm{C}$. Expression was then induced with $0.4 \mathrm{~m} M$ isopropyl $\beta$-D-1-thiogalactopyranoside (IPTG) at an $\mathrm{OD}_{600}$ of 1.0 and the cells were allowed to grow overnight. The cells were harvested by centrifugation at $6000 \mathrm{~g}$ and subsequently resuspended in buffer $A(50 \mathrm{~m} M$ Tris- $\mathrm{HCl} \mathrm{pH} 7.4$, $0.5 M \mathrm{NaCl}$ ) containing $20 \mathrm{~m} M$ imidazole, $0.1 \mathrm{mg} \mathrm{ml}^{-1}$ lysozyme, $0.05 \mathrm{mg} \mathrm{ml}^{-1}$ DNase and $2 \mathrm{mM} \mathrm{MgCl}$. After disrupting the cells with a high-pressure press (Constant Systems), the cell debris was removed by centrifugation at $50000 \mathrm{~g}$. The supernatant was then loaded onto a $5 \mathrm{ml}$ HisTrap HP column (GE Healthcare, USA) which had been pre-equilibrated with buffer $A$. Following sample loading, the column was washed with ten volumes of buffer $A$ before the target protein was eluted from the column with buffer $A$ supplemented with $200 \mathrm{~m} M$ imidazole. The eluted recombinant protein was desalted into buffer $B(10 \mathrm{~m} M$ Tris- $\mathrm{HCl} \mathrm{pH} 7.2,50 \mathrm{~m} M \mathrm{NaCl})$ using a PD-10 desalting column (GE Healthcare, USA) and the hexahistidine tag was then removed by incubating the sample with $1 \mathrm{U}$ thrombin (GE Healthcare, USA) per milligram of recombinant $\mathrm{IdmH}$ overnight at $4^{\circ} \mathrm{C}$. Thrombin was removed by loading the protein mixture onto a $5 \mathrm{ml}$ HiTrap Q column (GE Healthcare, USA) equilibrated with buffer $B$. The column was washed with five column volumes of buffer $B$ before a linear gradient of increasing $\mathrm{NaCl}$ concentration was applied to elute the bound proteins. $2.5 \mathrm{ml}$ fractions were collected across the gradient, which were analysed by SDSPAGE. Fractions containing the protein were pooled, concentrated and applied onto a size-exclusion chromatography column (HiLoad 26/600 Superdex 75 pg, GE Healthcare, USA) pre-equilibrated with buffer $B$. After a void volume of $40 \mathrm{ml}, 1 \mathrm{ml}$ fractions were collected. These were once more analysed using SDS-PAGE and the fractions containing the most highly pure IdmH were combined to form the final sample. This was concentrated to $20 \mathrm{mg} \mathrm{ml}^{-1}$ (as determined from the $A_{280}$ with an extinction coefficient of $17990 \mathrm{M}^{-1} \mathrm{~cm}^{-1}$ ) using a centrifugal filter concentration device (Vivaspin 20; 10000 molecular-weight cutoff). Finally, samples were flash-frozen in liquid nitrogen and stored at $-80^{\circ} \mathrm{C}$ until use. The IdmH- $\Delta 99-107$ variant was expressed and purified in exactly the same way.

Production of selenomethionine-labelled IdmH- $\Delta 99-107$ was achieved by cultivation of the methionine-auxotrophic E. coli strain B834 (DE3) cells in M9 minimal medium 
Table 1

Data-collection and refinement statistics.

Values in parentheses are for the highest resolution shell.

\begin{tabular}{|c|c|c|c|}
\hline & $\begin{array}{l}\text { Selenomethionine-labelled } \\
\text { IdmH- } \Delta 99-107\end{array}$ & $\begin{array}{l}\text { Native } \\
\text { IdmH- } \Delta 99-107\end{array}$ & Wild-type IdmH \\
\hline \multicolumn{4}{|l|}{ Data collection } \\
\hline Wavelength $(\AA ̊)$ & 0.966 & 0.976 & 0.9795 \\
\hline Space group & $F 23$ & $F 23$ & $P 12{ }_{1} 1$ \\
\hline$a, b, c(\AA)$ & $152.6,152.6,152.6$ & $152.7,152.7,152.7$ & $66.7,103.5,99.6$ \\
\hline$\alpha, \beta, \gamma\left({ }^{\circ}\right)$ & $90.0,90.0,90.0$ & $90.0,90.0,90.0$ & $90.0,91.6,90.0$ \\
\hline Resolution $(\AA)$ & $76.3-2.2(2.27-2.20)$ & $76.3-2.0(2.05-2.00)$ & $51.8-2.7(2.82-2.70)$ \\
\hline$R_{\text {merge }}$ & $0.118(4.620)$ & $0.046(0.669)$ & $0.086(0.961)$ \\
\hline$R_{\text {meas }}$ & $0.120(4.697)$ & $0.053(0.769)$ & $0.097(1.098)$ \\
\hline$R_{\mathrm{pim}}$ & $0.022(0.846)$ & $0.026(0.373)$ & $0.046(0.522)$ \\
\hline$\langle I / \sigma(I)\rangle$ & $20.8(1.4)$ & $12.7(1.4)$ & $11.4(1.4)$ \\
\hline $\mathrm{CC}_{1 / 2}$ & $0.999(0.812)$ & $0.999(0.516)$ & $0.998(0.656)$ \\
\hline Completeness (\%) & $100(100)$ & $99.9(99.9)$ & $99.6(99.5)$ \\
\hline Multiplicity & $30.6(30.6)$ & $4.1(4.1)$ & $4.5(4.3)$ \\
\hline \multicolumn{4}{|l|}{ Refinement } \\
\hline Resolution $(\AA)$ & $88.1-2.2$ & $88.2-2.0$ & $103.5-2.7$ \\
\hline No. of reflections (total/free) & $14989 / 726$ & 19980/974 & $37126 / 1832$ \\
\hline$R_{\text {work }} / R_{\text {free }}{ }^{\dagger}$ & $0.212 / 0.276$ & $0.230 / 0.271$ & $0.211 / 0.245$ \\
\hline Total No. of atoms & 3707 & 3704 & 19690 \\
\hline No. of ligands & 0 & 0 & 0 \\
\hline No. of water molecules & 13 & 27 & 9 \\
\hline Wilson $B$ factor $\left(\AA^{2}\right)$ & 48.3 & 48.4 & 60.7 \\
\hline \multicolumn{4}{|l|}{ R.m.s. deviations } \\
\hline Bond lengths $(\AA)$ & 0.016 & 0.007 & 0.012 \\
\hline Bond angles $\left({ }^{\circ}\right)$ & 1.81 & 1.15 & 1.47 \\
\hline \multicolumn{4}{|l|}{ Ramachandran plot } \\
\hline Favoured (\%) & 98.8 & 98.8 & 98.46 \\
\hline Allowed (\%) & 1.2 & 1.2 & 1.47 \\
\hline Outliers $(\%)$ & 0 & 0 & 0.07 \\
\hline Molecules in asymmetric unit & 2 & 2 & 10 \\
\hline PDB code & $6 \mathrm{hnl}$ & $6 \mathrm{hnm}$ & $6 \mathrm{hnn}$ \\
\hline
\end{tabular}

the wild-type enzyme. The crystals were flash-cooled in liquid nitrogen for data collection, this time in the absence of cryoprotectant. X-ray diffraction data for selenomethionine-labelled IdmHه99-107 were recorded on MASSIF-1 at the European Synchrotron Radiation Facility (Bowler et al., 2015, 2016; Svensson et al., 2015, 2018; Nurizzo et al., 2016), while native IdmH- $\Delta 99-107$ data were recorded on beamline I03 at Diamond Light Source.

All diffraction data were indexed and integrated using XDS (Kabsch, 2010) before subsequent scaling in AIMLESS and data processing using the $C C P 4 i 2$ graphical user interface to $C C P 4$ (Potterton et al., 2018). The structure of IdmH- $\Delta 99-107$ was determined to $2 \AA$ resolution by single-wavelength anomalous dispersion (SAD) phasing using the SHELX pipeline followed by density modification using Parrot and automated model building in Buccaneer. Iterative rounds of manual model building and refinement were then performed in Coot and REFMAC5, respectively (Cowtan, 2006, 2010, 2012; Murshudov et al., 2011; Sheldrick, 2010). supplemented with BME vitamins (Sigma-Aldrich) and $50 \mu \mathrm{g} \mathrm{ml}^{-1} \mathrm{~L}-(+)$-selenomethionine (Anatrace). All expression and purification steps were performed as for the native protein. Incorporation of selenomethionine was confirmed by LC/MS analysis.

\subsection{Protein crystallization, X-ray data collection and structure determination}

Initial crystallization conditions for full-length IdmH were identified with The JCSG Core Suites I-IV (Qiagen) using Formulatrix NT8 robotic mixing of protein at $20 \mathrm{mg} \mathrm{ml}^{-1}$ with crystallization solution at $1: 1,2: 1$ and $1: 2$ volumetric ratios in separate drops. IdmH crystal hit conditions were subsequently optimized with respect to $\mathrm{pH}$ and precipitant concentration using hanging-drop vapour diffusion, giving the crystals used for data collection in $0.2 \mathrm{M}$ calcium acetate, $0.1 \mathrm{M} \mathrm{MES} \mathrm{pH}$ $5.5,18.6 \%(w / v)$ polyethylene glycol 8000 . The crystals were cryoprotected by soaking them in mother-liquor solution containing $20 \%(v / v)$ glycerol for about $30 \mathrm{~s}$ before flashcooling them by plunging them into liquid nitrogen. X-ray diffraction data for full-length $\mathrm{IdmH}$ were collected on beamline I04 at Diamond Light Source.

The structure of IdmH could not be determined by molecular replacement using these data and the structure was therefore solved using selenomethionine-labelled IdmH- $\Delta 99$ 107. Crystals were obtained in 3.6-4 $M$ sodium formate, 10$15 \%(v / v)$ glycerol using the same approach as described for
The structure of wild-type $\mathrm{IdmH}$ was solved by molecular replacement using a single monomer of IdmH- $\Delta 99-107$ as a search model in Phaser (McCoy, 2007; McCoy et al., 2007). The final model was once more generated following iterative rounds of manual model building in Coot and refinement using REFMAC5 imposing local noncrystallographic symmetry (NCS) restraints throughout (Emsley \& Cowtan, 2010; Murshudov et al., 2011). The model was validated using MolProbity (Chen et al., 2010). All data-collection and structure-refinement statistics are listed in Table 1.

\subsection{NMR spectroscopy}

$950 \mathrm{MHz}$ Bruker Ascend Aeon and $750 \mathrm{MHz}$ Oxford NMR spectrometers equipped with cryogenically cooled tripleresonance probes were used for all NMR experiments. Spectra

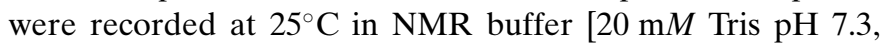
$50 \mathrm{~m} M \mathrm{NaCl}, 1 \mathrm{~m} M$ DTT, $\left.0.02 \%(w / v) \mathrm{NaN}_{3}, 5 \%(v / v) \mathrm{D}_{2} \mathrm{O}\right]$. 2D NMR experiments were carried out using Wilmad NMR tubes (Precision, limit $600 \mathrm{MHz}$ frequency), while Shigemi NMR tubes were used for triple-resonance experiments.

2.4.1. Backbone nuclei resonance assignment. Triplelabelled protein was obtained by growing E. coli BL21(DE3) cells containing the wild-type IdmH expression plasmid in $\mathrm{M} 9$ medium prepared in $\mathrm{D}_{2} \mathrm{O}$ and supplemented with $\left[{ }^{13} \mathrm{C}\right]-\mathrm{D}$ glucose and ${ }^{15} \mathrm{NH}_{4} \mathrm{Cl}$ (Cambridge Isotope Laboratories). Labelled protein was purified as described for the wild type, except that no thrombin-cleavage step was carried out. A set 
of 3D BEST-TROSY backbone resonance assignment spectra [HNCA, $\mathrm{HN}(\mathrm{CO}) \mathrm{CA}, \mathrm{HNCO}, \mathrm{HN}(\mathrm{CA}) \mathrm{CB}$ and $\mathrm{HN}(\mathrm{CO})$ CACB] were recorded at $750 \mathrm{MHz}$, while a 3D BEST-TROSY $\mathrm{HN}(\mathrm{CA}) \mathrm{CO}$ spectrum was recorded at $950 \mathrm{MHz}$, using pulse sequences (Solyom et al., 2013) obtained from the Institut de Biologie Structurale (IBS), Grenoble, France for $\left[{ }^{15} \mathrm{~N},{ }^{13} \mathrm{C},{ }^{2} \mathrm{H}\right]$ labelled IdmH at a concentration of $0.8 \mathrm{mM}$. Non-uniform sampling was used to speed up the acquisition time and obtain high-resolution spectra.

The raw spectra were pre-processed with NMRPipe and NMRDraw (Delaglio et al., 1995). Non-uniform sampled data were reconstructed using the $M d d N M R$ suite (Jaravine \& Orekhov, 2006; Jaravine et al., 2008). Backbone nuclei resonance assignment was performed using CCPNmr Analysis (Vranken et al., 2005) and AutoAssign (Zimmerman et al., 1997).

2.4.2. Chemical shift perturbation studies. ${ }^{15} \mathrm{~N}$-labelled protein was prepared by growing E. coli strain BL21(DE3) containing the wild-type idmH expression plasmid in M9 minimal medium using ${ }^{15} \mathrm{NH}_{4} \mathrm{Cl}$ as the sole nitrogen source. ${ }^{1} \mathrm{H}-{ }^{15} \mathrm{~N}$ HSQC-TROSY spectra were collected at an IdmH concentration of $0.2 \mathrm{mM}$ with varying concentrations of indanomycin $(0-200 \mu \mathrm{M})$ at $25^{\circ} \mathrm{C}$ at a field strength of $750 \mathrm{MHz}$. The raw data were pre-processed using TopSpin (Bruker). The NMR spectra were analysed using CCPNmr Analysis (Vranken et al., 2005). The minimal chemical shift perturbation was calculated from ${ }^{1} \mathrm{H}$ and ${ }^{15} \mathrm{~N}$ chemical shift differences using 0.154 as a scaling factor for ${ }^{15} \mathrm{~N}$ shift changes and calculating the closest chemical shift distance between peaks in the spectra with and without indanomycin (Williamson, 2013).

\subsection{In silico modelling}

Molecular docking of indanomycin (1) and an analogue truncated at C8 was performed using AutoDock Vina 1.1.2 (Trott \& Olson, 2010). AutoDockTools 1.5 .4 was used to merge the nonpolar hydrogens and define all formally single bonds as rotatable bonds for docking. Subsequently, docking was performed with AutoDock Vina using a grid of $25 \times 20 \times$ $25 \AA$ centred on the active-site cavity (in either chain $A$ or chain $B$ of the crystal structure). Three top-scoring poses in different orientations were selected by filtering out highly similar poses and poses that did not place the indane ring inside the cavity. Subsequently, the protein-product poses (with the complete indanomycin) were prepared for molecular-dynamics simulation with AmberTools17 (Case et al., 2017) using the Enlighten protocols (see https://github.com/ marcvanderkamp/enlighten). For one of the poses, a different hydrogen-bonding network was obtained by 'flipping' the Asn117 side chain. Simulations were thus performed on four different initial protein-product complexes. The PREP protocol was used to prepare the protein-ligand system for simulation, which involved (i) adding hydrogens [all residues in their standard protonation states in line with prediction by PROPKA 3.1 (Olsson et al., 2011; Søndergaard et al., 2011); His44 singly protonated on $\mathrm{H}^{\delta 1}$ with all other His singly protonated on $\mathrm{H}^{\varepsilon 2}$ as predicted by the AmberTools program reduce], (ii) parameterization of indanomycin using AM1BCC partial charges and GAFF parameters assigned by Antechamber (Wang et al., 2004, 2006) and (iii) solvation of the active site with TIP3P water molecules (in a $20 \AA$ sphere centred on the $\mathrm{N}$ atom of indanomycin, in addition to crystallographically determined water molecules). After a brief structural optimization (using the STRUCT protocol, which involves simulated annealing and energy minimization), a molecular-dynamics simulation of 200 or $300 \mathrm{ps}$ was performed for each pose (using the DYNAM protocol at a constant temperature of $298 \mathrm{~K}$ ).

Snapshots from molecular-dynamics simulations between 100 and $300 \mathrm{ps}$ were used to start approximate QM/MM umbrella sampling simulations of the reverse Diels-Alder reaction (at least $10 \mathrm{ps}$ apart) using the same protocol as used previously (Byrne et al., 2016) using DFTB2 (Elstner et al., 1998) for indanomycin (QM region) and the Amber force field ff14SB (Maier et al., 2015) for the enzyme environment. In short, the reaction coordinate used to follow the Diels-Alder reaction was the distance between the centre of mass of the dienophile carbons ( $\mathrm{C} 19$ and $\mathrm{C} 20)$ and the centre of mass of the diene carbons to which they bond (C15 and C12). By using this reaction-coordinate definition, no bias is applied as to which carbon-carbon bond is formed or broken first (or indeed whether or not the bonds are formed/broken synchronously). Umbrella sampling was performed from reaction coordinate value 1.2 to value $3.8 \AA$ (in steps of $0.1 \AA$, with additional windows at $2.15,2.25$ and $2.35 \AA$ ). The same conditions were used as in the DYNAM protocol, apart from using a $1 \mathrm{fs}$ timestep (instead of $2 \mathrm{fs}$ ). Snapshots were saved every 0.5 ps. A restraint of $100 \mathrm{kcal} \mathrm{mol}^{-1} \AA^{-1}$ and 2 ps of simulation was used for each umbrella sampling window. Reaction-coordinate values were recorded every 1 fs and used for input to the Weighted-Histogram Analysis Method (WHAM; Grossfield, 2013), resulting in the potential of mean force (free energy) along the reaction coordinate (bin width of $0.05 \AA$ ). For each docking pose, 11 QM/MM reaction simulations were performed, each starting from a different snapshot. The QM/MM protocols are designed to limit computational resources and are thus approximate. Our aim is not to analyse the reaction in detail, but to distinguish between different possible reactive binding poses. For example, the use of a single, combined reaction coordinate (obtaining a 1D-PMF) is less accurate than a 2D-PMF with sampling along two reaction coordinates representing the carbon-carbon bonds formed [see, for example, Świderek \& Moliner (2016), where this is discussed in detail]. With the combined reaction coordinate used here, reasonable sampling is obtained along the minimum free-energy region indicated in a 2D-PMF (see Supplementary Fig. S3). Although longer sampling (for example 20 ps per window after a 2 ps equilibration) affects the free-energy barriers for simulations, we have tested that this does not significantly change the differences in the barrier between different poses (similar to as reported by Hirvonen et al., 2019). To obtain representative snapshots for the approximate transition state, the centroid of the highest 
populated cluster was used, as obtained with hierarchical agglomerative clustering on the r.m.s.d. of the indanomycin atoms after alignment on the protein backbone on the structures sampled in the windows with reaction-coordinate restraints at 2.2 and $2.3 \AA$.

\section{Results}

3.1. Structural determination of IdmH- $\Delta 99-107$ and native IdmH

The N-terminally hexahistidine-tagged wild-type IdmH was successfully expressed in E. coli strain BL21(DE3) cells and purified to homogeneity, and the hexahistidine tag was removed by thrombin cleavage. Size-exclusion chromatography and LC/MS analysis (Supplementary Fig. S4) suggested a dimeric oligomeric state for this protein. Initial attempts at crystallization yielded small needle-like crystals, but optimization of these initial conditions produced larger rod-shaped crystals. X-ray diffraction data were collected to $2.7 \AA$ resolution, but attempts to determine the structure by molecular replacement were unsuccessful, presumably owing to the lack of a suitable search model and the large number of molecules in the asymmetric unit as estimated using MATTPROB (Kantardjieff \& Rupp, 2003).

To improve crystallization, a number of variants were produced to reduce the surface entropy (Goldschmidt et al., 2007) or to truncate predicted surface loops (Roy et al., 2010; Supplementary Fig. S1 and Table S1). One variant, containing a deletion of residues 99-107, resulted in large, easily reproducible crystals in the cubic space group $F 23$ [Supplementary Fig. S5(b)] from new crystallization conditions. This protein was also produced labelled with selenomethionine and crystallized under identical conditions, allowing the crystal structure of IdmH- $\Delta 99-107$ to be determined to $2 \AA$ resolution using selenium SAD phasing. The asymmetric unit comprises

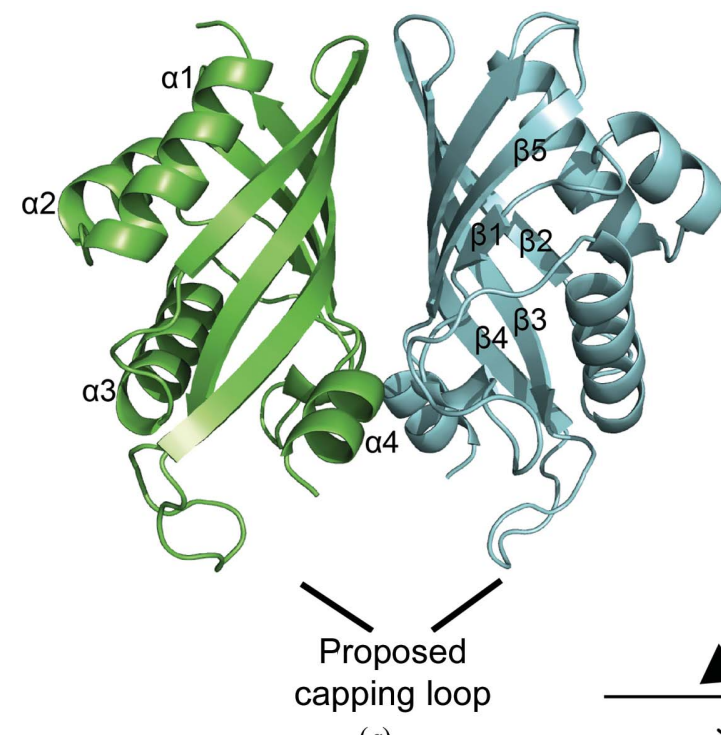

(a)

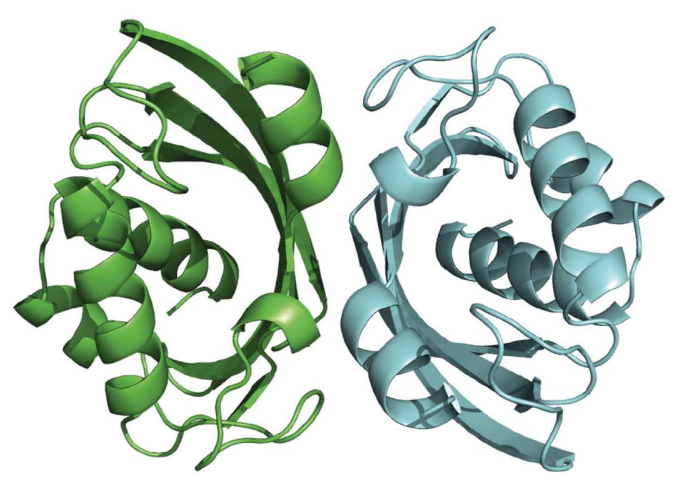

(c)

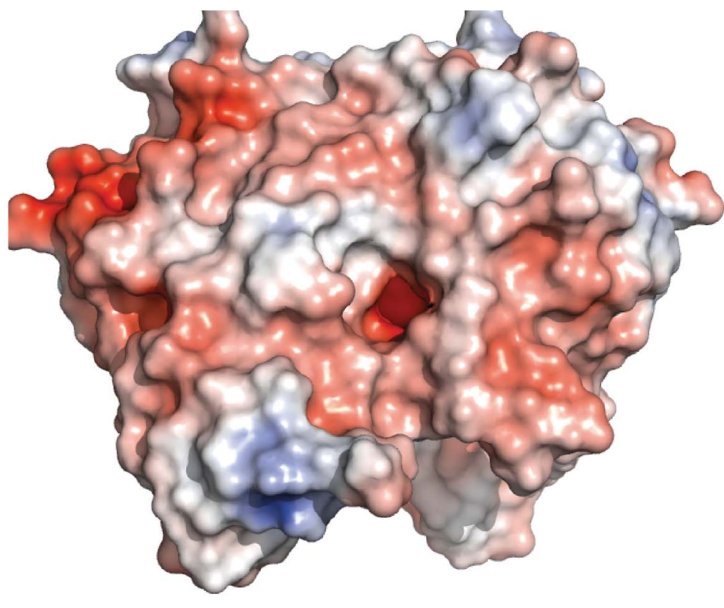

$90^{\circ}$

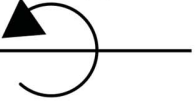

(b)

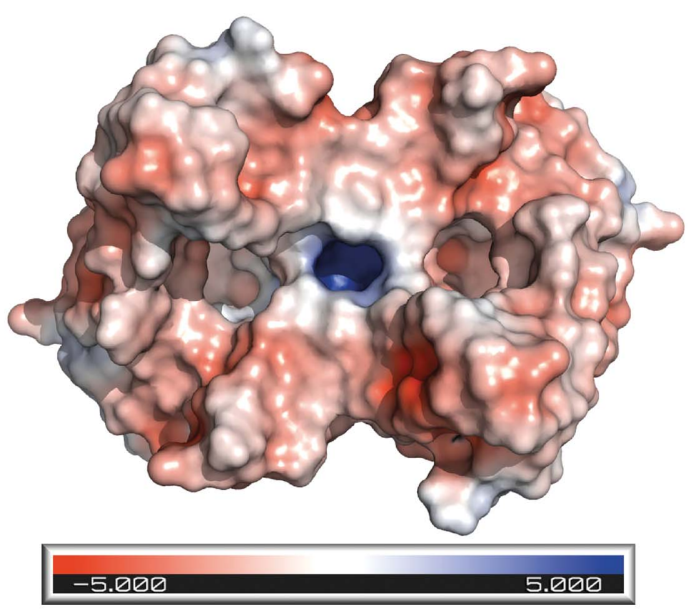

$(d)$

Figure 2

The structure of IdmH. $(a, c)$ Ribbon diagrams showing the IdmH dimer architecture. Individual monomers are coloured green and blue, respectively. The overall shape of the monomer can be described as a distorted $\alpha / \beta$-barrel with the dimerization interface located between the $\beta$-sheets. Each monomer comprises four $\alpha$-helices $(\alpha 1-\alpha 4)$ and five $\beta$-strands $(\beta 1-\beta 5)$. $(b, d)$ Surface representation of the IdmH dimer. The surface is coloured using APBS (Jurrus et al., 2018) according to the approximate electrostatic potential from $-5 k T \mathrm{e}^{-1}$ (red) to $5 k T \mathrm{e}^{-1}$ (blue). 
one copy of the IdmH- $\Delta 99-107$ dimer with the entire polypeptide chain successfully modelled into density, except for the $\mathrm{N}$ - and $\mathrm{C}$-terminal regions. Both the selenomethioninelabelled protein structure and the isomorphous native structure of IdmH- $\Delta 99-107$ were modelled independently (Table 1), giving almost identical structures that superpose with an r.m.s.d. of $0.12 \AA$ over 257 amino acids. The remainder of the structural description will focus on the unlabelled native protein as this should best represent the natural form of the enzyme. With this structure in hand, the crystal structure of wild-type IdmH was determined by molecular replacement using a single subunit of IdmH- $\Delta 99-107$ as a search model (Fig. 2). Electron-density maps were well defined for all ten protomers in the asymmetric unit, allowing all chains to be modelled, with the exception of some residues at the extreme $\mathrm{N}$ - and C-termini (residues in the ranges 1-6 and 143-148). The final model was refined at $2.7 \AA$ resolution with an $R_{\text {work }}$. of 0.21 and an $R_{\text {free }}$ of 0.24 . Both models were checked with MolProbity, revealing a high-quality model (MolProbity scores of 1.00 and 0.91 for wild-type IdmH and IdmH- $\Delta 99$ 107 , respectively) with no Ramachandran outliers.

\subsection{Analysis of the IdmH structure}

3.2.1. The overall fold. The ten molecules in the asymmetric unit for the wild-type protein form five almost identical dimers. The following discussion of the $\operatorname{IdmH}$ structure will therefore focus on the dimer formed by chains $A$ and $B$ unless otherwise stated, as this represents the most well ordered dimer based on an analysis of the overall $B$ factors of the chains. The structure of IdmH reveals a homodimer, with individual protomers consisting of a core $\alpha+\beta$ barrel. Each subunit is composed of a five-stranded mixed $\beta$-sheet with four strands $(\beta 2-\beta 5)$ continuous in sequence and an additional $\beta$-strand $(\beta 1)$, considerably shorter in length, provided later in the polypeptide chain (Fig. 2). The $\beta$-sheet of each subunit is curved and forms the central part of a distorted $\alpha+\beta$ barrel. The barrel is completed by three $\alpha$-helices $(\alpha 1-\alpha 3)$ on the periphery of the dimer and a fourth short helix $(\alpha 4)$ completing the fold. The dimer assembly is driven by hydrophobic interactions between the $\beta$-sheets from the two subunits, generating the dimer shown in Fig. 2.

At the centre of each protomer, a large hydrophobic pocket is found which is proposed to form the IdmH active site (see below). Two loops (residues 38-46 and 99-108) are located near to the entrance of this hydrophobic pocket and limit the access to the proposed active site from the bulk solvent. Interestingly, the loop, which comprises residues $99-108$ and is partially truncated in the $\mathrm{IdmH}-\Delta 99-107$ variant, appears to occupy two distinct conformations within each of the five dimers in the asymmetric unit. Indeed, in each dimer each protomer has this loop occupying either a more open or a more closed conformation above the hydrophobic cavity. Whilst we cannot rule this out as an artefact of the crystallization, this suggests that this loop is likely to be highly dynamic in solution and may also hint at a potential change in conformation between the two protomers, suggesting the potential for allostery in this enzyme (Supplementary Fig. S6). Furthermore, studies of a number of Diels-Alderases have shown that a loop covering the active site becomes more ordered upon substrate binding and this loop could also play such a role here (Byrne et al., 2016; Zheng et al., 2016).

3.2.2. The proposed active site. A deep hydrophobic pocket penetrating towards the core of the barrel can be observed in each IdmH protomer. It is likely that this constitutes the active site of the enzyme (Fig. 3 and Supplementary Fig. S7). Each pocket is lined by the side chains of residues Tyr16, Phe19, Leu39, Trp59, Val62, Trp63, Met115, His129, Ser133 and Asn135. We propose that the preponderance of hydrophobic residues might act to steer the hydrophobic substrate (4 in Fig. 1) into an appropriate conformation for facile ring cyclization by acting as a natural product template, as observed by others in structurally related cyclases (Barajas et al., 2017; Li et al., 2010; Liu et al., 2015; Carey \& Sundberg, 2007).

3.2.3. Sequence and structural homologues of $\mathrm{IdmH}$. BLASTp (Supplementary Fig. S2) and DALI searches against the PDB revealed that the closest sequence and structural homologue to $\mathrm{IdmH}$ is the putative polyketide cyclase from Chromobacterium violaceum (PDB entry 4 lgq; $30 \%$ sequence identity; Joint Center for Structural Genomics, unpublished work). Many of the closest structural and sequence matches, however, stem from structural genomics efforts and thus lack accompanying biochemical data and publications supporting their functional annotation. The putative hydrolases AcIR (PDB entry 2gey) and SnoaL2 (PDB entry 2gex) from $S$. galilaeus and S. nogalater, respectively (Beinker et al., 2006) as well as the polyketide cyclase SnoaL (PDB entry 1sjw; Sultana et al., 2004) did appear as close structural matches to the structure of IdmH even though they share at best $28 \%$ sequence identity. We therefore focused our structural

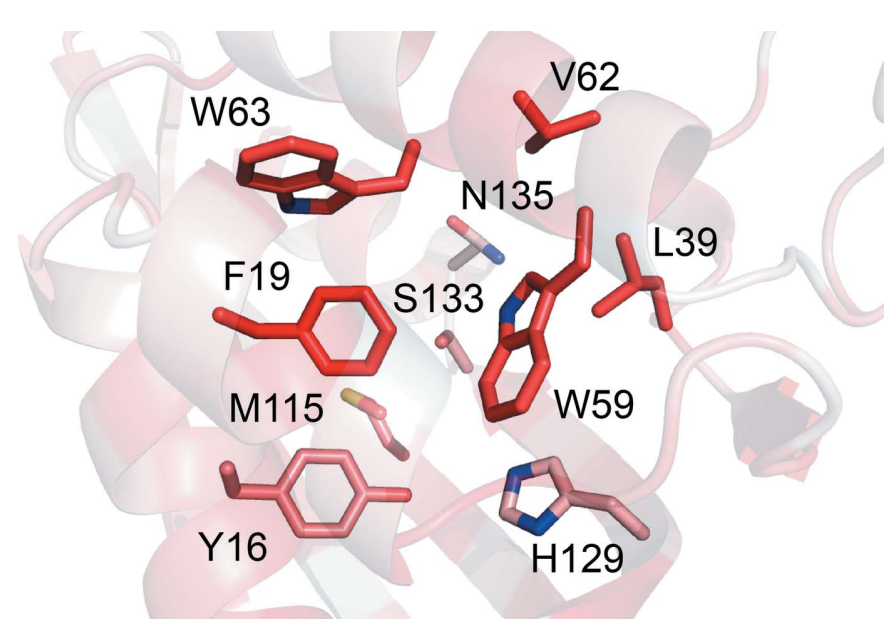

Figure 3

Detailed view of the putative IdmH active site, highlighting key residues. The abundance of aromatic/hydrophobic residues in the active site might play a role in the catalysis of indane-ring formation by guiding the mainly hydrophobic substrate into an appropriate conformation for catalysis. The residues are coloured according to the normalized consensus hydrophobicity scale, where the most hydrophobic residues are red and the most hydrophilic residues are white (Eisenberg et al., 1984). 
comparisons against these biochemically characterized enzymes.

We superimposed the structure of IdmH on its sequence homologues using PyMOL (Schrödinger) and found that the polyketide cyclase SnoaL from the nogalamycin biosynthetic pathway (PDB entry 1sjw; Sultana et al., 2004) exhibited the highest structural similarity to $\mathrm{IdmH}$ as measured by the rootmean-square deviation of the fit (r.m.s.d. $=1.56 \AA$ over 549 atoms) [Fig. 4(a)]. Crystallographic analysis and gel-filtration chromatography experiments both suggest that SnoaL forms a tetramer in solution (Sultana et al., 2004), in contrast to IdmH which is homodimeric. SnoaL catalyses an intramolecular aldol condensation using the conserved residue Asp121 as an $\mathrm{acid} / \mathrm{base}$ catalyst during the reaction. Other essential residues for the activity of SnoaL include Asn33, Gln105 and two histidines, His107 and His119. In contrast, the active site of IdmH does not contain residues that can perform acid/base chemistry at equivalent positions to the catalytic Asp121 and His107 of SnoaL, but rather contains Thr131 and Asn117 instead [Fig. 4(b)]. Of note, Thr131 is implicated as important in the suggested catalytic mechanism of IdmH discussed later. Both active sites include many aromatic residues, and some of them are conserved between the two proteins (for example Phe19 in IdmH and Phe15 in SnoaL and His129 in IdmH and His119 in SnoaL). There are also two tryptophan residues (Trp63 in IdmH and Trp54 in SnoaL) that appear to be conserved between these proteins; however, they appear to be in entirely different orientations. These different orientations could reflect genuine differences between these two structures or they may arise from the fact that the SnoaL structure contains a bound substrate. Furthermore, there are additional tryptophan (Trp59) and tyrosine (Tyr16) residues in IdmH which seem to be unique to this protein (SnoaL contains Tyr31 and Met11 at these locations) [Fig. 4(b)]. Taken together, these data suggest that $\mathrm{IdmH}$ is unlikely to catalyze an aldol condensation as found for SnoaL and is likely to interact with its substrate in a different manner.

\subsection{Investigating IdmH enzymatic activity by nuclear magnetic resonance spectroscopy}

We had no access to the potential IdmH substrate $\mathbf{4}$, so we chose to investigate the binding of the product, indanomycin (1), to the enzyme by 2D nuclear magnetic resonance (NMR) spectroscopy. Although this did not allow us to directly test the hypothesis that IdmH catalyses indane-ring formation, we postulated that the enzyme would bind the product of the

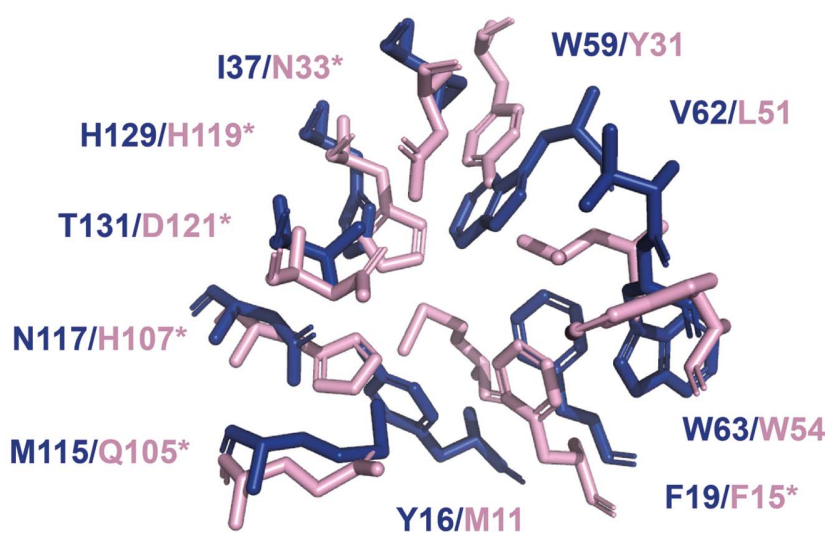

(b)

Comparison of IdmH (blue) with its sequence and structural homologue SnoaL (pink). (a) IdmH chain $A$ was aligned with SnoaL (PDB entry 1sjw) chain $A$ using PyMOL. (b) Active-site residues in SnoaL (pink) the two pro different choices and positioning of aromatic residues (Trp59/Tyr31 and Trp63/Trp54), while Tyr16 appears reaction, providing evidence to support its role in indanomycin biosynthesis.

IdmH was labelled with ${ }^{13} \mathrm{C},{ }^{15} \mathrm{~N}$ and ${ }^{2} \mathrm{H}$ and, following the collection and processing of a number of triple-resonance spectra (Yamazaki et al., 1994; Supplementary Table S2), the NMR resonances of backbone nuclei were assigned for $88 \%$ of residues. To investigate indanomycin binding, $\left[{ }^{15} \mathrm{~N}\right]-\mathrm{IdmH}$ was mixed with a stoichiometric amount of ligand (indanomycin) and a ${ }^{1} \mathrm{H}-{ }^{15} \mathrm{~N}$ HSQC-TROSY spectrum was acquired (Fig. 5). Significant chemical shift perturbations (CSPs) for a number of backbone amides compared with the native protein were seen on addition of indanomycin, suggesting a binding event.

To investigate the binding further and to localize the indanomycin-binding site, titration spectra with substoichiometric ligand:protein ratios were recorded. Significant shifts in both the ${ }^{1} \mathrm{H}$ and ${ }^{15} \mathrm{~N}$ spectra were observed for a number of the assigned residues (see Supplementary Fig. S8 for examples of specific residue chemical shift perturbations during the titration). Since shifts can be influenced by through-bond, throughspace and allosteric interactions, it is difficult to determine whether the CSPs in IdmH are a result of direct binding or conformational change. However, to localize the proteinligand interactions, the geometrical distance moved by each peak was calculated for all assigned residues [Fig. 6(a)] and chemical shifts larger than the standard deviation of all shifts were mapped onto the IdmH diagram using yellow, orange and red colours [Fig. 6(b)].

In the presence of indanomycin (the bound spectrum), some resonances moved a substantial distance from their resonance counterparts in the native protein (the free spectrum) making it impossible to match the two accurately. For this reason, a 'minimum chemical shift procedure' was employed (Farmer et al., 1996; Lüttgen et al., 2002; Williamson, 2013). This method links each assigned free resonance to the signal in the bound spectrum that has moved the least from the position in the free spectrum. Therefore, each resonance is 
assigned a CSP, and while the true change might be larger than that assigned, it will never be smaller.

The results of chemical shift mapping suggest that the most significant CSPs are localized at the top of the hydrophobic cavity of $\operatorname{IdmH}(\alpha$-helices $3-4)$ and on the $\beta$-sheet inside the pocket $(\beta$ strands 2-4) [Figs. 2 and 6(c)], consistent with the predicted location of the IdmH active site. A closer look at the activesite residues [Fig. $6(d)$ ] revealed that the majority of the resonances exhibited a moderate (Val62 and Met115) to large (Trp59, Trp63 and Tyr16) shift. Two resonances, Ser133 and Ile37, exhibited a shift below the level of significance (<0.72 p.p.m.) and three remaining resonances, Thr131, Phe19 and His129, do not have their backbone resonances assigned. These results thus suggest that indanomycin can bind to $\mathrm{IdmH}$ in the proposed active site, and that binding, and potentially catalysis, could induce conformational changes which spread to other areas of the enzyme, as highlighted by the more distal CSPs.

In a bid to obtain further details of indanomycin binding to $\mathrm{IdmH}$, we performed both co-crystallization and crystal-soaking experiments to try and determine a structure of the product complex. Co-crystallization with the ligand appeared to inhibit crystal formation, likely owing to the high concentration of DMSO required to keep the ligand in solution, whilst crystal soaking did not result in significant new electron density that could be confidently attributed to the ligand. In the light of these results, we therefore elected to explore computational methods as a means of further analyzing the biochemical function of $\mathrm{IdmH}$.

\subsection{Docking the product and simulating the protein-product complex}

Having determined that indanomycin binds in the active site of $\mathrm{IdmH}$, we wanted to further explore the likely chemical reaction catalysed by $\mathrm{IdmH}$. Indanomycin was placed in the wild-type $\operatorname{IdmH}$ active sites (chain $A$ and $B$ ) using in silico molecular docking. Three significantly different binding poses were found (two in chain $A$ and one in chain $B$ ). For the binding pose in chain $B$, the conformer for Asn117 was adjusted to provide a slight difference in the hydrogenbonding environment. This led to the following four options: pose $\mathrm{A}$, the indane ring bound in the chain $A$ pocket but without any specific hydrogen bonds being made; pose $\mathrm{B}$, the indane ring in the chain $A$ pocket with a hydrogen bond formed between Ser133 and the substrate carbonyl group $(\mathrm{C} 21=\mathrm{O})$; pose $\mathrm{C}$, the indane ring in the chain $B$ pocket with a hydrogen bond formed between Ser133 and the substrate carbonyl group $(\mathrm{C} 21=\mathrm{O})$ but in a different orientation to pose B (Fig. 8); pose D, the indane ring in the chain $B$ pocket with a hydrogen bond formed between Thr131 and the substrate carbonyl group in which a new hydrogen-bond network is formed in the protein between Asn117 and Tyr16. Poses C and D are shown in Fig. 7 (after brief structural optimization), with the main difference in binding pose arising from the side-chain orientation of Asn117. Subsequently, brief structural optimization and 100-300 ps molecular-dynamics simulation was performed for each of the four poses.

\section{5. $\mathrm{QM} / \mathrm{MM}$ simulations of the reverse reaction}

$11 \mathrm{QM} / \mathrm{MM}$ reaction simulations (starting from independent snapshots of the molecular-dynamics simulations) were run for all four possible enzyme-substrate complexes using the same approximate protocol as described in Byrne et al. (2016). Representative (approximate) transition-state structures of these four complexes are shown in Fig. 8, together with the free-energy profile obtained from the 11 simulations together. Based on the energy barriers, reactions proceeding from starting poses $\mathrm{C}$ and $\mathrm{D}$ are the most likely (Fig. 7). The low barriers compared with poses $\mathrm{A}$ and $\mathrm{B}$ can be rationalized based on the electron-withdrawing nature of the hydrogen bond formed between Ser133 or Thr131 and the substrate carbonyl at C21 (depending on the pose). Between the two, pose $\mathrm{D}$ seems to be the most likely, based on a good fit with the 
cavity and some possible catalytic stabilization by the positioning of Trp59. The reaction barrier for option A is highest, which could be expected owing to the lack of a (good) hydrogen-bonding interaction in this pose; during the QM/ MM simulations a hydrogen bond to Trp59 can be formed (see
Fig. 8), but this interaction is more transient and thus does not offer the same benefit as the hydrogen bonds formed in poses $\mathrm{C}$ and $\mathrm{D}$. MD and QM/MM simulation of option B lead to a poorer fit with the active-site cavity, suggesting that this is an unlikely starting point for catalysis by IdmH (Fig. 8).

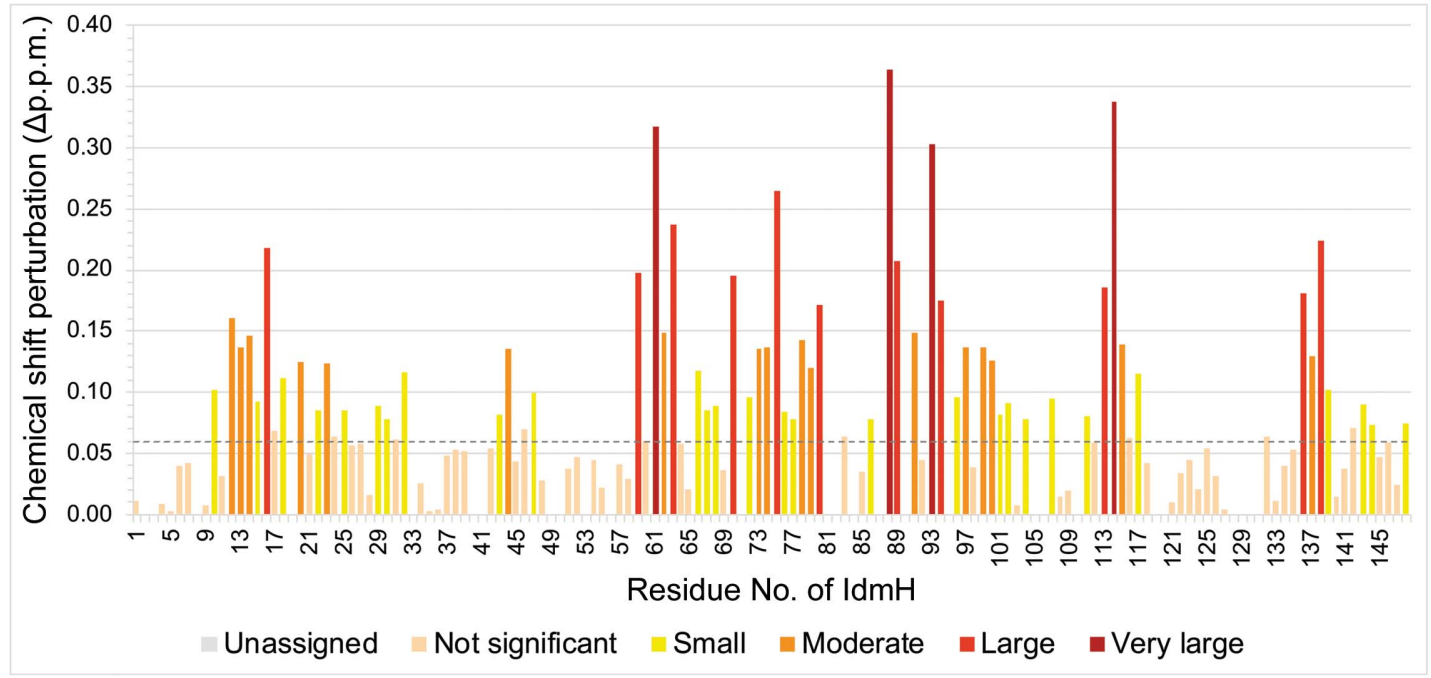

(a)
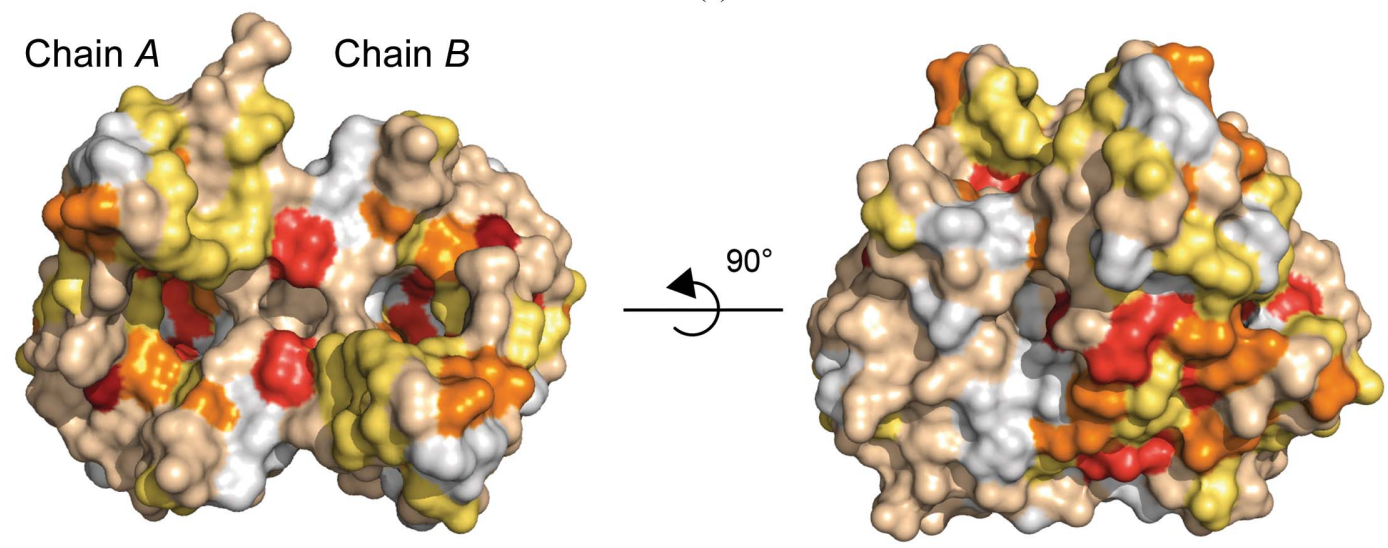

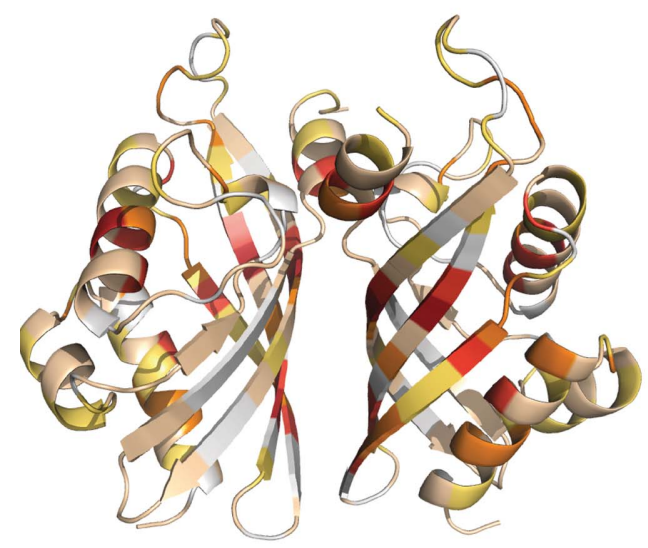

(c)

(b)

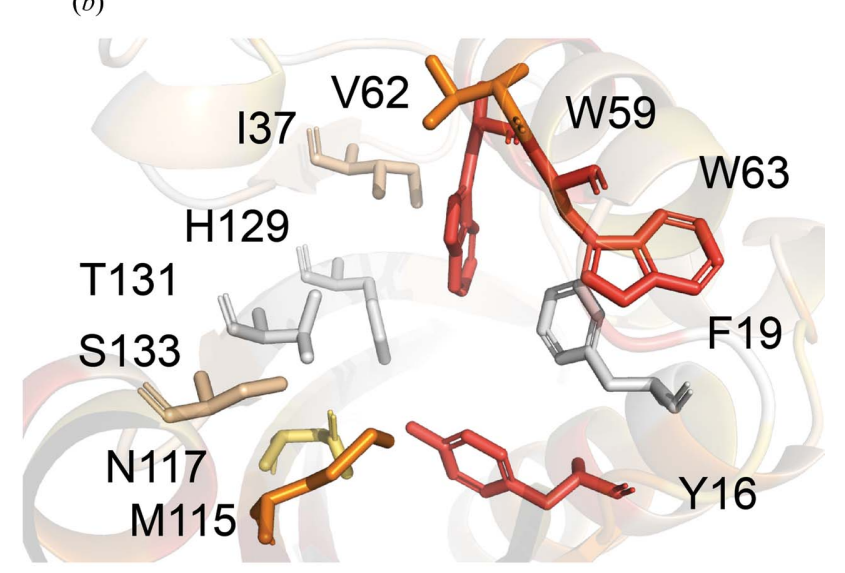

$(d)$

Figure 6

Chemical shift perturbations (CSPs) mapped onto IdmH following the addition of indanomycin. (a) Histogram showing the geometrical distance moved by each peak assigned to a residue. Peaks are coloured from yellow to dark red as the value of the CSP increases. Chemical shifts which were smaller than the standard deviation of all shifts (0.072 p.p.m.) are coloured pale cream. Surface $(b)$ and ribbon $(c)$ representations of IdmH with peak shifts mapped using the same colour scheme as in $(a)$. Residues which could not be assigned in the NMR spectrum are coloured white. $(d)$ Active-site cavity of chain $B$ with the proposed catalytic residues coloured according to the same colour scheme as in $(b)$ and $(c)$. 
The chemical shift mapping results (Fig. 6) further support the observations from the approximate $\mathrm{QM} / \mathrm{MM}$ simulations. Pose D involves a hydrogen-bonding network between Thr131, Asn117 and Tyr16 as well as catalytic stabilization from Trp59. According to the NMR titration experiments, all of these residues (except for Thr131, which could not be measured by significant CSPs (0.22 p.p.m. for Tyr16, 0.12 p.p.m. for Asn117 and 0.20 p.p.m. for Trp59). There are also van der Waals interactions between the substrate and the other residues with significant CSPs. It is further worth noting that Ser133, which is involved in a hydrogen bond to the substrate in pose C, displayed a CSP (0.012 p.p.m.) below the level of significance ( 0.07 p.p.m.), making pose $\mathrm{C}$ less likely than pose $\mathrm{D}$. The reaction simulations indicate that an asynchronous concerted mechanism is followed, with the formation of the $\mathrm{C} 15-\mathrm{C} 19$ bond occurring first (and repreassigned) are involved in protein-ligand interactions as

senting the reaction barrier; see Fig. 8); this is similar to the situation in the intramolecular Diels-Alder reaction in AbyU and indeed the uncatalysed reaction (Byrne et al., 2016).

In summary, chemical shift perturbation mapping, in silico docking and approximate $\mathrm{QM} / \mathrm{MM}$ simulations reveal the structure of the likely reactive IdmH-substrate complex and show that the active-site shape, along with a critical hydrogen bond, is likely to promote the correct conformation of the substrate 4 (bringing together the diene and dienophile) and provides an appropriate environment for the indane-ring cyclization reaction to occur.

\section{Discussion}

The Diels-Alder reaction is of major synthetic value in organic chemistry for the preparation of substituted sixmembered rings with the creation of up to four new stereocenters (Stocking \&
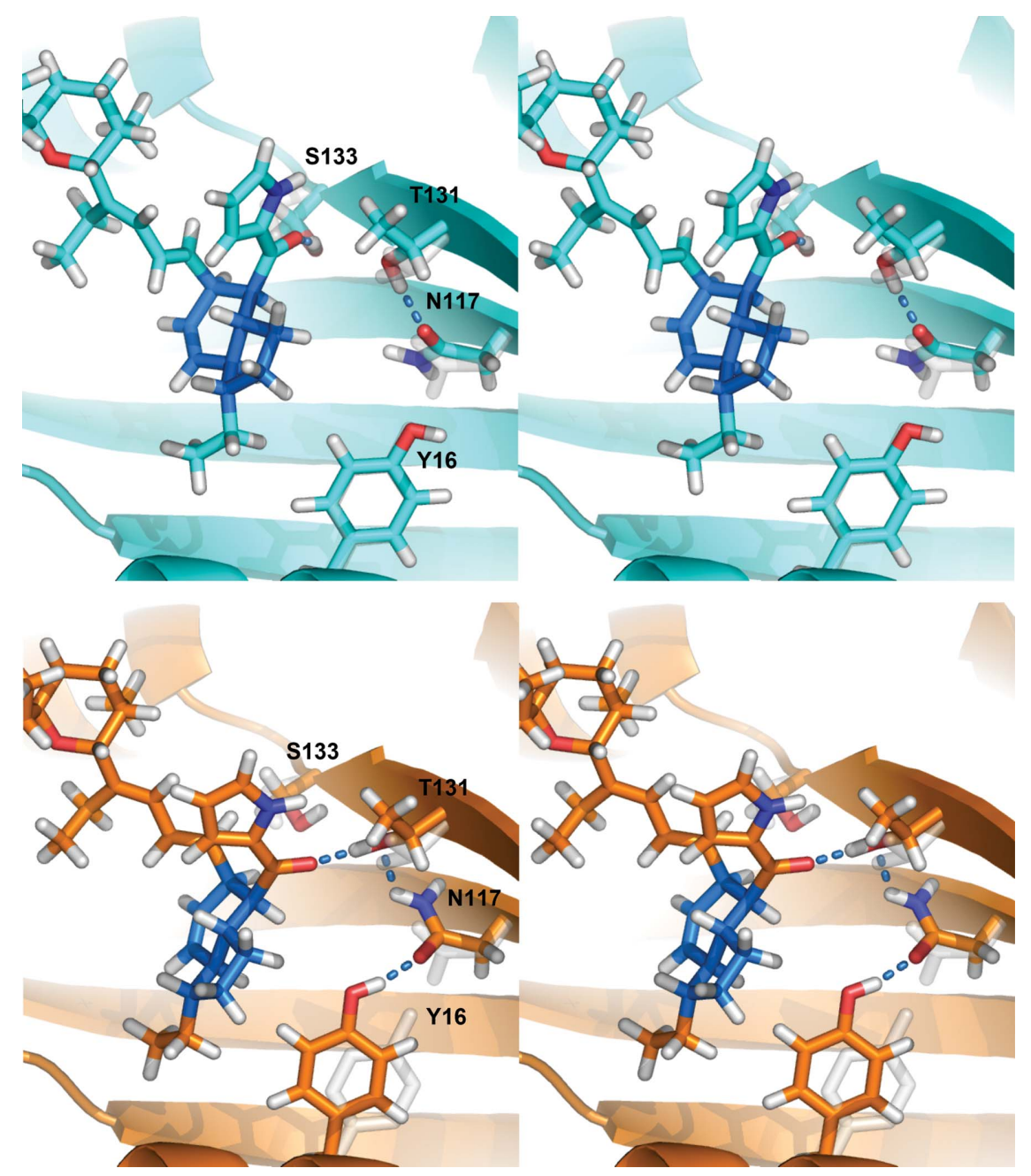

Figure 7

Stereoview of the position and interactions of indanomycin docked in the wild-type IdmH chain $B$ active site after brief structural optimization. Top: pose $\mathrm{C}$, indanomycin $\mathrm{C} 21=\mathrm{O}$ hydrogen-bonds to Ser133. Bottom: pose $\mathrm{D}$, indanomycin $\mathrm{C} 21=\mathrm{O}$ hydrogen-bonds to Thr131. The Tyr16, Asn117 and Thr131 side-chain positions from the original crystal structure are shown transparently in light grey. Williams, 2003). The potential use of proteins to catalyse Diels-Alder reactions stereoselectively under mild conditions therefore remains of high interest. Until recently, no natural enzymes that specifically catalyse Diels-Alder reactions (i.e. DielsAlderases) were known (Jamieson et al., 2019), and some progress was made with obtaining catalytic antibodies and de novo designed enzymes that catalyse a bimolecular Diels-Alder reaction (Gouverneur et al., 1993; Siegel et al., 2010). During the late 1990s and early 2000s, three putative DielsAlderases involved in natural product synthesis were reported: macrophomate synthase (Watanabe et al., 2000), lovastatin nonaketide synthase (LovB; Auclair et al., 2000) and solanapyrone synthase (Sol5; Katayama et al., 1998; Kasahara et al., 2010). LovB and Sol5 have activities other than catalysing a Diels-Alder reaction, which makes proving any catalysis of the cycloaddition reaction difficult. The bimolecular reaction catalysed by macrophomate synthase is now thought to follow a stepwise mechanism: Michael addition followed by an aldol reaction (Guimarães et al., 2005). In the 2010s, the first enzyme specifically catalysing only a Diels-Alder reaction was reported: SpnF, which is involved in the biosynthesis of spinosyn A (Kim et al., 2011). Subsequently, numerous standalone Diels-Alderases have been identified, especially from bacterial biosynthesis pathways, including the cofactor-free PyrI4 (Zheng et al., 2016) from the pyrroindomycin biosynthetic pathway and its homologues AbyU (Byrne et al., 2016) and VstJ 
(Hashimoto et al., 2015) from other spirotetronate-containing natural products.

Here, we propose that IdmH from the biosynthesis of indanomycin is another natural standalone Diels-Alderase. Similar to the structurally characterized natural DielsAlderases AbyU (Byrne et al., 2016) and PyrI4 (Zheng et al., 2016), the enzyme acts in the (final) tailoring steps of natural product biosynthesis, does not require a cofactor and the reaction catalysed is intermolecular. The IdmH structure is a homodimeric 'barrel' that contains a 'capping loop'. With the capping loop closed, a cavity is formed that fits the substrate in a conformation in which the diene and dienophile moieties are positioned in line with a Diels-Alder reaction. Interestingly, the structure and sequence of IdmH are substantially different from those of AbyU and PyrI4 (as well as their homologues from other spirotetronate and spirotetramate biosynthesis pathways; Supplementary Fig. S9). Instead of a $\beta$-barrel, $\operatorname{IdmH}$ is an $\alpha+\beta$ barrel. There is no significant sequence similarity between IdmH on the one hand and AbyU and PyrI4 on the other, nor is there an obvious mapping of the four antiparallel $\beta$-strands to the four antiparallel $\beta$-strands of the AbyU and PyrI $\beta$-barrels. Despite the absence of a clear evolutionary relationship between the $\operatorname{IdmH} \alpha+\beta$ barrel and the $\beta$-barrels of $\mathrm{AbyU}$ and PyrI4, the overall shape is similar, with the substrate cavity near the capping loop in each case (Supplementary Fig. S9; Hashimoto \& Kuzuyama, 2016). This raises the question of whether the emergence of these intramolecular Diels-Alderases with similar structures provide an
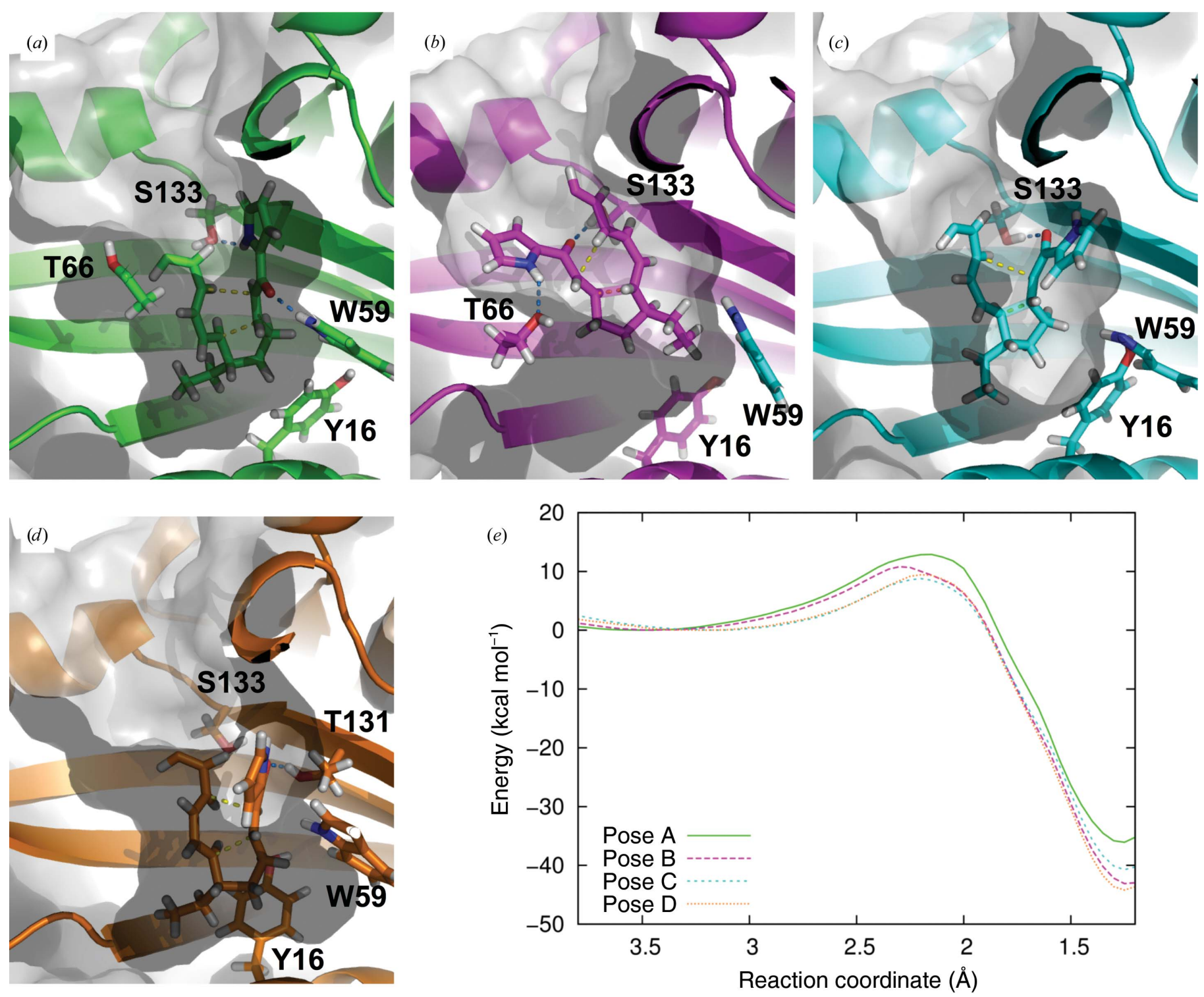

Figure 8

The results of approximate QM/MM reaction simulations (DFTB2/ff14SB). (a)-(d) show representative (approximate) transition-state structures of the four possible enzyme-product complexes (obtained by clustering) together with their energy profiles in $(e)$. Only part of the indanomycin structure is shown in these panels for clarity, but the remainder of the indanomycin molecule (including the tetrahydropyran ring, which may or may not be cyclized before IdmH-catalysed formation of the indane ring), was present in all simulations. 
example of convergent evolution. Further, it indicates that not only a $\beta$-barrel scaffold, but also a $\alpha+\beta$ barrel scaffold, could be used for the design of engineered or de novo designed Diels-Alderases, thereby potentially allowing a broader range of substrates.

\section{NMR data}

The NMR assignment data for IdmH have been deposited in the Biological Magnetic Resonance Data Bank (BioMagResBank) with accession number 27838.

\section{Acknowledgements}

We thank Diamond Light Source for access to beamlines I03 and I04 (MX15378) and the European Synchrotron Radiation Facility for access to MASSIF-1 (MX1828) that contributed to the results presented here. NMR spectroscopy was carried out at the Astbury Biostructure Laboratory using $950 \mathrm{MHz}$ Bruker Ascend Aeon and $750 \mathrm{MHz}$ Oxford NMR magnets (University of Leeds ABSL award and Wellcome Trust 104920/Z/14/Z). We thank Dr Anastasia Zhuravleva (University of Leeds) and Dr Gary Thompson (University of Kent) for their help in acquiring and processing 3D NMR spectra, and Dr Ryan Seipke and Dan Van (University of Leeds) for helpful discussions during this work. The simulation work was conducted using the computational facilities of the Advanced Computing Research Centre of the University of Bristol.

\section{Funding information}

ID was supported by the Wellcome Trust (105214/Z/14/Z). MWvdK and GRH were supported by BBSRC David Phillips Fellowships (BB/M026280/1) to Marc W. van der Kamp and (BB/N019970/1) to Glyn R. Hemsworth.

\section{References}

Auclair, K., Sutherland, A., Kennedy, J., Witter, D. J., Van den Heever, J. P., Hutchinson, C. R. \& Vederas, J. C. (2000). J. Am. Chem. Soc. 122, 11519-11520.

Barajas, J. F., Shakya, G., Moreno, G., Rivera, H., Jackson, D. R., Topper, C. L., Vagstad, A. L., La Clair, J. J., Townsend, C. A., Burkart, M. D. \& Tsai, S.-C. (2017). Proc. Natl Acad. Sci. USA, 114, E4142-E4148.

Beinker, P., Lohkamp, B., Peltonen, T., Niemi, J., Mäntsälä, P. \& Schneider, G. (2006). J. Mol. Biol. 359, 728-740.

Bowler, M. W., Nurizzo, D., Barrett, R., Beteva, A., Bodin, M., Caserotto, H., Delagenière, S., Dobias, F., Flot, D., Giraud, T., Guichard, N., Guijarro, M., Lentini, M., Leonard, G. A., McSweeney, S., Oskarsson, M., Schmidt, W., Snigirev, A., von Stetten, D., Surr, J., Svensson, O., Theveneau, P. \& MuellerDieckmann, C. (2015). J. Synchrotron Rad. 22, 1540-1547.

Bowler, M. W., Svensson, O. \& Nurizzo, D. (2016). Crystallogr. Rev. 22, 233-249.

Byrne, M. J., Lees, N. R., Han, L.-C., van der Kamp, M. W., Mulholland, A. J., Stach, J. E. M., Willis, C. L. \& Race, P. R. (2016). J. Am. Chem. Soc. 138, 6095-6098.

Carey, F. A. \& Sundberg, R. J. (2007). Advanced Organic Chemistry. Part B: Reactions and Synthesis, 5th ed. New York: Springer.
Case, D. A., Cerutti, D. S., Cheatham, T. E., Darden, T. A., Duke, R. E., Giese, T. J., Gohlke, H., Götz, A. W., Greene, D., Homeyer, N., Izadi, S., Kovalenko, A., Lee, T. S., Le Grand, S., Li, P., Lin, C., Liu, J., Luchko, T., Luo, R., Mermelstein, D., Merz, K. M., Monard, G., Nguyen, H., Omelyan, I., Onufriev, A., Pan, F., Qi, R., Roe, D. R., Roitberg, A. E., Sagui, C., Simmerling, C. L., Botello-Smith, W. M., Swails, J. M., Walker, R. C., Wang, J., Wolf, R. M., Wu, X., Xiao, L., York, D. M. \& Kollman, P. A. (2017). AMBER 2017. University of California, San Francisco.

Chen, V. B., Arendall, W. B., Headd, J. J., Keedy, D. A., Immormino, R. M., Kapral, G. J., Murray, L. W., Richardson, J. S. \& Richardson, D. C. (2010). Acta Cryst. D66, 12-21.

Cowtan, K. (2006). Acta Cryst. D62, 1002-1011.

Cowtan, K. (2010). Acta Cryst. D66, 470-478.

Cowtan, K. (2012). Acta Cryst. D68, 328-335.

Cummings, M., Breitling, R. \& Takano, E. (2014). FEMS Microbiol. Lett. 351, 116-125.

Delaglio, F., Grzesiek, S., Vuister, G. W., Zhu, G., Pfeifer, J. \& Bax, A. (1995). J. Biomol. NMR, 6, 277-293.

Dutton, C. J., Banks, B. J. \& Cooper, C. B. (1995). Nat. Prod. Rep. 12, 165-181.

Edwards, M. P., Ley, S. V., Lister, S. G., Palmer, B. D. \& Williams, D. J. (1984). J. Org. Chem. 49, 3503-3516.

Eisenberg, D., Schwarz, E., Komaromy, M. \& Wall, R. (1984). J. Mol. Biol. 179, 125-142.

Elstner, M., Porezag, D., Jungnickel, G., Elsner, J., Haugk, M., Frauenheim, T., Suhai, S. \& Seifert, G. (1998). Phys. Rev. B, 58, 7260-7268.

Emsley, P., Lohkamp, B., Scott, W. G. \& Cowtan, K. (2010). Acta Cryst. D66, 486-501.

Farmer, B. T., Constantine, K. L., Goldfarb, V., Friedrichs, M. S., Wittekind, M., Yanchunas, J., Robertson, J. G. \& Mueller, L. (1996). Nat. Struct. Biol. 3, 995-997.

Goldschmidt, L., Cooper, D. R., Derewenda, Z. S. \& Eisenberg, D. (2007). Protein Sci. 16, 1569-1576.

Gouverneur, V. E., Houk, K. N., de Pascual-Teresa, B., Beno, B., Janda, K. D. \& Lerner, R. A. (1993). Science, 262, 204208.

Grossfield, A. (2013). WHAM: The Weighted Histogram Analysis Method v.2.0.9.1. http://membrane.urmc.rochester.edu/content/ wham.

Guimarães, C. R., Udier-Blagović, M. \& Jorgensen, W. L. (2005). J. Am. Chem. Soc. 127, 3577-3588.

Ha, N.-C., Choi, G., Choi, K. Y. \& Oh, B.-H. (2001). Curr. Opin. Struct. Biol. 11, 674-678.

Hashimoto, T., Hashimoto, J., Teruya, K., Hirano, T., Shin-ya, K., Ikeda, H., Liu, H., Nishiyama, M. \& Kuzuyama, T. (2015). J. Am. Chem. Soc. 137, 572-575.

Hashimoto, T. \& Kuzuyama, T. (2016). Curr. Opin. Chem. Biol. 35, 117-123.

Hirvonen, V. H. A., Hammond, K., Chudyk, E. I., Limb, M. A. L., Spencer, J., Mulholland, A. \& van der Kamp, M. W. (2019). J. Chem. Inf. Model. 59, 3365-3369.

Jamieson, C. S., Ohashi, M., Liu, F., Tang, Y. \& Houk, K. N. (2019). Nat. Prod. Rep. 36, 698-713.

Jaravine, V. A. \& Orekhov, V. Y. (2006). J. Am. Chem. Soc. 128, 13421-13426.

Jaravine, V. A., Zhuravleva, A. V., Permi, P., Ibraghimov, I. \& Orekhov, V. Y. (2008). J. Am. Chem. Soc. 130, 3927-3936.

Jurrus, E., Engel, D., Star, K., Monson, K., Brandi, J., Felberg, L., Brookes, D., Wilson, L., Chen, J., Liles, K., Chun, M., Li, P., Gohara, D., Dolinsky, T., Konecny, R., Koes, D., Nielsen, J., Head-Gordon, T., Geng, W., Krasny, R., Wei, G., Holst, M., McCammon, J. \& Baker, N. (2018). Protein Sci. 27, 112-128.

Kabsch, W. (2010). Acta Cryst. D66, 133-144.

Kallio, P., Sultana, A., Niemi, J., Mäntsälä, P. \& Schneider, G. (2006). J. Mol. Biol. 357, 210-220.

Kantardjieff, K. A. \& Rupp, B. (2003). Protein Sci. 12, 1865-1871. 
Kasahara, K., Miyamoto, T., Fujimoto, T., Oguri, H., Tokiwano, T., Oikawa, H., Ebizuka, Y. \& Fujii, I. (2010). Chembiochem, 11, 12451252.

Katayama, K., Kobayashi, T., Oikawa, H., Honma, M. \& Ichihara, A. (1998). Biochim. Biophys. Acta, 1384, 387-395.

Kim, H. J., Ruszczycky, M. W., Choi, S.-H., Liu, Y.-N. \& Liu, H. (2011). Nature (London), 473, 109-112.

Li, C., Roege, K. E. \& Kelly, W. L. (2009). Chembiochem, 10, 1064 1072.

Li, Y., Xu, W. \& Tang, Y. (2010). J. Biol. Chem. 285, 22764-22773.

Liu, L., Zhang, Z., Shao, C.-L., Wang, J.-L., Bai, H. \& Wang, C.-Y. (2015). Sci. Rep. 5, 10463.

Lundqvist, T., Rice, J., Hodge, C. N., Basarab, G. S., Pierce, J. \& Lindqvist, Y. (1994). Structure, 2, 937-944.

Lüttgen, H., Robelek, R., Mühlberger, R., Diercks, T., Schuster, S. C., Köhler, P., Kessler, H., Bacher, A. \& Richter, G. (2002). J. Mol. Biol. 316, 875-885.

Maier, J. A., Martinez, C., Kasavajhala, K., Wickstrom, L., Hauser, K. E. \& Simmerling, C. (2015). J. Chem. Theory Comput. 11, 36963713.

McCoy, A. J. (2007). Acta Cryst. D63, 32-41.

McCoy, A. J., Grosse-Kunstleve, R. W., Adams, P. D., Winn, M. D., Storoni, L. C. \& Read, R. J. (2007). J. Appl. Cryst. 40, 658-674.

Minami, A., Ose, T., Sato, K., Oikawa, A., Kuroki, K., Maenaka, K., Oguri, H. \& Oikawa, H. (2014). ACS Chem. Biol. 9, 562-569.

Murshudov, G. N., Skubák, P., Lebedev, A. A., Pannu, N. S., Steiner, R. A., Nicholls, R. A., Winn, M. D., Long, F. \& Vagin, A. A. (2011). Acta Cryst. D67, 355-367.

Newman, D. J. \& Cragg, G. M. (2012). J. Nat. Prod. 75, 311-335.

Nurizzo, D., Bowler, M. W., Caserotto, H., Dobias, F., Giraud, T., Surr, J., Guichard, N., Papp, G., Guijarro, M., Mueller-Dieckmann, C., Flot, D., McSweeney, S., Cipriani, F., Theveneau, P. \& Leonard, G. A. (2016). Acta Cryst. D72, 966-975.

Olano, C., Méndez, C. \& Salas, J. A. (2010). Nat. Prod. Rep. 27, 571616.

Olsson, M. H., Søndergaard, C. R., Rostkowski, M. \& Jensen, J. H. (2011). J. Chem. Theory Comput. 7, 525-537.

Potterton, L., Agirre, J., Ballard, C., Cowtan, K., Dodson, E., Evans, P. R., Jenkins, H. T., Keegan, R., Krissinel, E., Stevenson, K., Lebedev, A., McNicholas, S. J., Nicholls, R. A., Noble, M., Pannu, N. S., Roth, C., Sheldrick, G., Skubak, P., Turkenburg, J., Uski, V., von Delft, F., Waterman, D., Wilson, K., Winn, M. \& Wojdyr, M. (2018). Acta Cryst. D74, 68-84.
Rommel, K. R., Li, C. \& Kelly, W. L. (2011). Org. Lett. 13, 2536-2539.

Roy, A., Kucukural, A. \& Zhang, Y. (2010). Nat. Protoc. 5, 725-738. Sheldrick, G. M. (2010). Acta Cryst. D66, 479-485.

Siegel, J. B., Zanghellini, A., Lovick, H. M., Kiss, G., Lambert, A. R., St Clair, J. L., Gallaher, J. L., Hilvert, D., Gelb, M. H., Stoddard, B. L., Houk, K. N., Michael, F. E. \& Baker, D. (2010). Science, 329, 309-313.

Solyom, Z., Schwarten, M., Geist, L., Konrat, R., Willbold, D. \& Brutscher, B. (2013). J. Biomol. NMR, 55, 311-321.

Søndergaard, C. R., Olsson, M. H., Rostkowski, M. \& Jensen, J. H. (2011). J. Chem. Theory Comput. 7, 2284-2295.

Stocking, E. M. \& Williams, R. M. (2003). Angew. Chem. Int. Ed. 42, 3078-3115.

Sultana, A., Kallio, P., Jansson, A., Wang, J.-S., Niemi, J., Mäntsälä, P. \& Schneider, G. (2004). EMBO J. 23, 1911-1921.

Svensson, O., Gilski, M., Nurizzo, D. \& Bowler, M. W. (2018). Acta Cryst. D74, 433-440.

Svensson, O., Malbet-Monaco, S., Popov, A., Nurizzo, D. \& Bowler, M. W. (2015). Acta Cryst. D71, 1757-1767.

Świderek, K. \& Moliner, V. (2016). J. Phys. Chem. B, 120, $2053-$ 2070.

Tang, L., Ward, S., Chung, L., Carney, J. R., Li, Y., Reid, R. \& Katz, L. (2004). J. Am. Chem. Soc. 126, 46-47.

Trott, O. \& Olson, A. J. (2010). J. Comput. Chem. 31, 455-461.

Vranken, W. F., Boucher, W., Stevens, T. J., Fogh, R. H., Pajon, A., Llinas, P., Ulrich, E. L., Markley, J. L., Ionides, J. \& Laue, E. D. (2005). Proteins, 59, 687-696.

Wang, J., Wang, W., Kollman, P. A. \& Case, D. A. (2006). J. Mol. Graph. Model. 25, 247-260.

Wang, J., Wolf, R. M., Caldwell, J. W., Kollman, P. A. \& Case, D. A. (2004). J. Comput. Chem. 25, 1157-1174.

Watanabe, K., Mie, T., Ichihara, A., Oikawa, H. \& Honma, M. (2000). J. Biol. Chem. 275, 38393-38401.

Williamson, M. P. (2013). Prog. Nucl. Magn. Reson. Spectrosc. 73, 116.

Yamazaki, T., Lee, W., Arrowsmith, C. H., Muhandiram, D. R. \& Kay, L. E. (1994). J. Am. Chem. Soc. 116, 11655-11666.

Zheng, Q., Guo, Y., Yang, L., Zhao, Z., Wu, Z., Zhang, H., Liu, J., Cheng, X., Wu, J., Yang, H., Jiang, H., Pan, L. \& Liu, W. (2016). Cell Chem. Biol. 23, 352-360.

Zimmerman, D. E., Kulikowski, C. A., Huang, Y., Feng, W., Tashiro, M., Shimotakahara, S., Chien, C.-Y., Powers, R. \& Montelione, G. T. (1997). J. Mol. Biol. 269, 592-610. 